\title{
The effects of irregularities and misalignments of the guide strings on stress levels in selected load-bearing elements of a conveyance
}

\author{
Stanisław Wolny $^{1, *}$, and Agata Drzewosz ${ }^{1, *}$ \\ ${ }^{1}$ AGH university of Science and Technology, Kraków, Poland
}

\begin{abstract}
In order that the ultimate state methods can be applied in dimensioning of the load-bearing elements in a conveyance it is required that their design loads should be determined during their normal duty cycle and under the emergency braking conditions.

This study aims to verify the predicted loading conditions obtained by theoretical analyses through experiments on a real object. Prior to the actual experimental procedure, limited to measurements of stress acting in the loadbearing cables in the conveyance, the system parameters have been identified. Special attention was given to parameters of roller guides and random irregularities of the guiding strings, which give rise to horizontal displacements of the conveyance and generate the conveyance- shaft steelwork interaction forces.
\end{abstract}

\section{Introduction}

With the assumption made that horizontal displacements of lumped masses are induced by random irregularities and misalignments of the guide strings[1,2], the analysis of the skip's dynamic behaviour in normal operating conditions (at fixed hoisting velocity $V_{0}=$ const) would yield the spectral densities of interaction forces between modelled masses as well as variances of the force amplitudes.

For the adopted calculation model of the skip (a 2D framework diagram), the magnitudes of forces were established that act upon the cross-sections of the load-bearing cables. In the work [3] the bending moments were determined. Thus obtained values of forces acting upon the cable cross-sections allowed the maximal stress levels acting upon a single cable to be determined [3].

The adequacy of the theoretical analyses of system dynamics and of fatigue endurance aspects has been verified through experiments on a real object $[4,5]$, involving stress and strain measurements in cables. Measurement results are compiled in [6].

The analysis of measurement results reveals a major discrepancy between predicted data and experimental results. The study is limited to comparing and contrasting experimental results with predicated data including:

- conveyance-shaft steelwork interaction forces

*Corresponding author: stwolny@agh.edu.pl,drzewosz@agh.edu.pl 
- $\quad$ stress levels in load-bearing cables in the conveyance.

Underlying the theoretical analyses are the following assumptions made:

- physical parameters of the guiding elements (roller guides) are based on the manufacturer's specifications;

- horizontal displacements of modelled masses are induced by irregularities and misalignments of the guide strings.

In order to find the cause of such major discrepancy of results, the Authors first identified the real parameters of roller guides and examined the actual irregularities of the guiding strings.

\section{Roller guides are the components of the investigated hoist installation}

The schematic diagram of the hoist installation considered in the experimental procedure is shown in Fig 1.

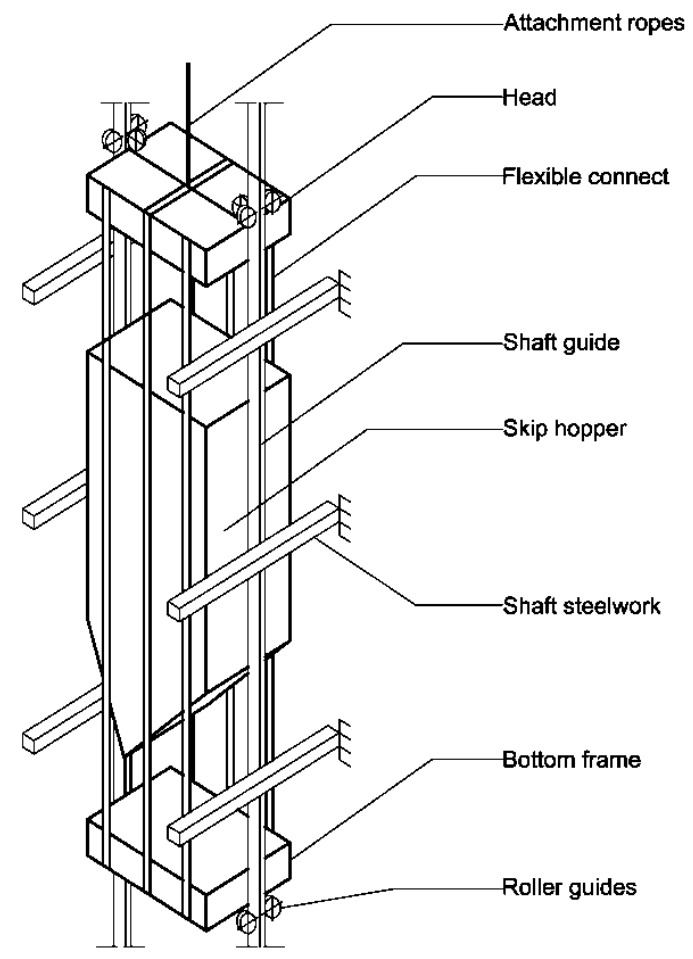

Fig 1. Schematic diagram of the hoist installation [7].

Fig 2 shows the structural design of the guide element mounted on the conveyance [7]. 


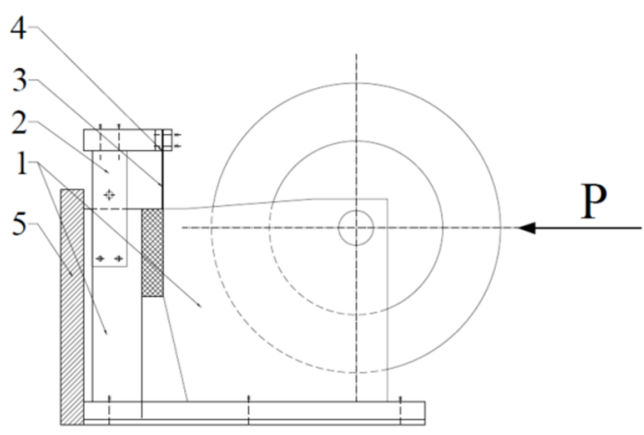

Fig 2. Diagram of the front and lateral guides (1- roller guide, 2- body of the measuring instrument,3elastic bar, 4- strain gauge, 5-base)[7].

Elasticity factor and viscous damping coefficient of the roller guides were obtained in laboratory tests performed as a part of the research study [7]. These parameters are:

$$
\mathrm{k}=7,5 \cdot 10^{5}\left[\frac{\mathrm{N}}{\mathrm{m}}\right], \quad 2 h=800\left[\frac{\mathrm{N} \cdot \mathrm{s}}{\mathrm{m}}\right] .
$$

Elasticity of the skip structure in the plane determined by the guides' front surface is derived from the formula [4]:

$$
k_{\mathrm{g}}=\frac{96 \mathrm{EI}_{1}}{\left(\frac{1}{2}\right)^{3}}
$$

$\mathrm{I}_{1}=\frac{\mathrm{bh}^{3}}{12}$ - inertia moment of the cable with respect to the main, central axis of inertia, perpendicular to the front plane of the skip;

- $\quad b, h[\mathrm{~m}]$ - dimensions of the cable cross- section;

- $\quad l_{1}$ - distance between the front guides on top and at the bottom frame.

Assuming the geometric dimensions of the conveyance to be [4]: $b=0,03 \mathrm{~m}, h=0,25 \mathrm{~m}$, $l_{1}=16 \mathrm{~m}$, we obtain:

$$
k_{\mathrm{g}}=\frac{96 \cdot 2,1 \cdot 10^{11} \cdot 0,03 \cdot 0,25^{3}}{12 \cdot 8^{3}}=1,57 \cdot 10^{6}\left[\frac{\mathrm{N}}{\mathrm{m}}\right] .
$$

\section{Irregularities of the guiding strings}

Irregularities and misalignments of the guiding strings in the hoistway were investigated in the shaft in which the experiment was carried out, involving the measurement of conveyance-shaft steelwork interaction forces and of stress levels in loadbearing elements of a conveyance $[4,6]$. Guiding strings, shown schematically in Fig 1, exhibit irregularities and certain misalignment, which were the subject of analysis. These irregularities of two guiding strings in the hoistway are designated as $\mathrm{fx}_{1}$ and $\mathrm{fx}_{4}$ (for one guide string) and $\mathrm{fx}_{2}$ and $\mathrm{fx}_{3}$ (for the other guide string). Measured misalignments of the guide string are plotted in Fig 3 and 4 . Fig 3 plots measured misalignment $\mathrm{fx}_{2}$ and $\mathrm{fx}_{3}$ (deflection from the vertical) for one guiding string, misalignments of the second string are shown in Fig 4. 


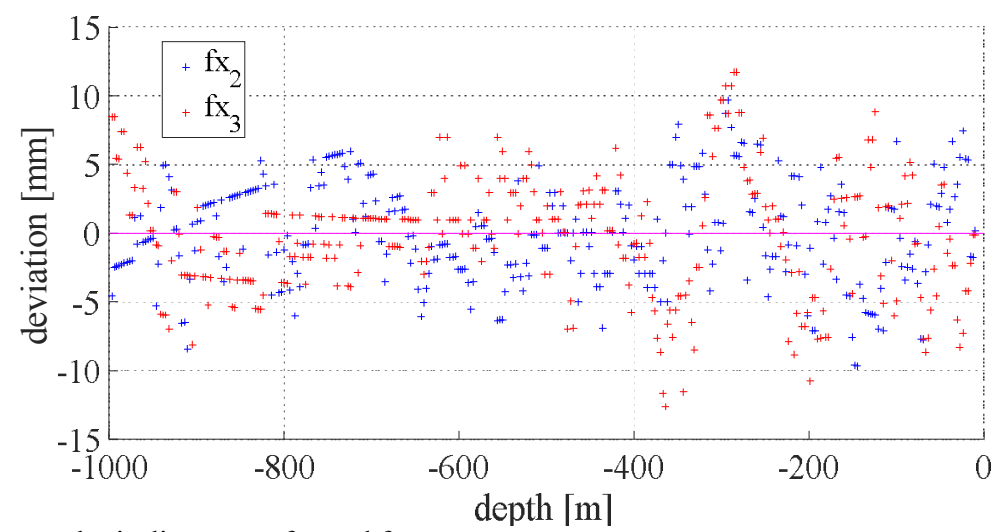

Fig. 3. Measured misalignments $\mathrm{fx}_{2}$ and $\mathrm{fx}_{3}$.

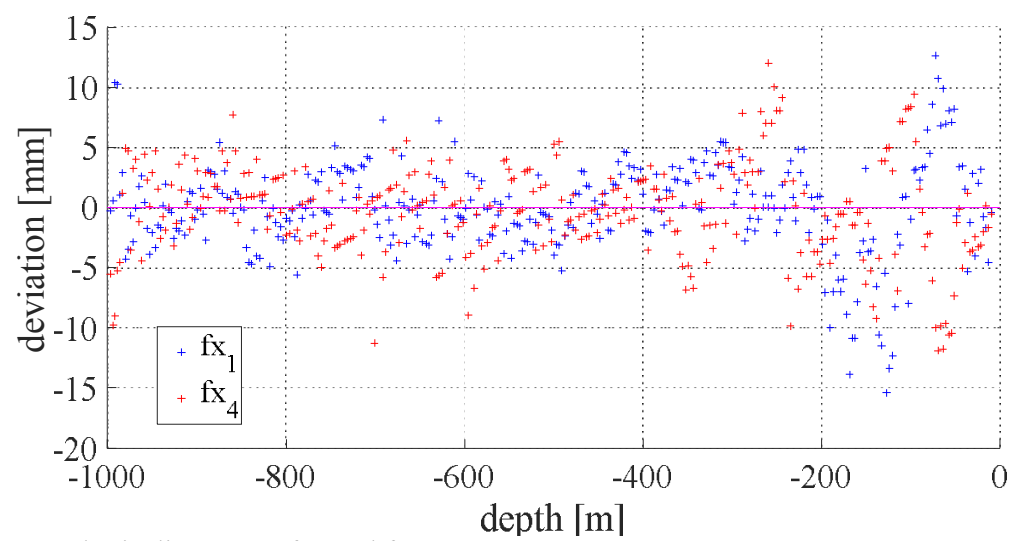

Fig. 4. Measured misalignments $\mathrm{fx}_{1}$ and $\mathrm{fx}_{4}$.

Measurement data were used to determine the function that correlates the irregularities of the guiding strings for varied hoisting velocities, given by the formula [8]:

$$
R(\tau)=\int_{-\infty}^{\infty} f(t) \bar{f}(t-\tau) d t
$$

where: $\mathrm{f}(\mathrm{t})$ - the original signal, $\bar{f}(t-\tau)$ - a correlated signal delayed by $\tau$, $R(\tau)$ - correlation function

Calculating the correlation functions of irregularities and misalignments of the guide strings, we proceed to obtain the spectral density functions of those irregularities (see Fig 5, $6,7)$.

Spectral density functions capturing guide string irregularities are plotted separately for each of the guide rails, for three values of hoisting velocity. The maximal values range from $1,5 \mathrm{mms}^{2} \div 4 \mathrm{mms}^{2}$. A thorough analysis of these plots reveals a certain dependence associated with the actual graph shape. By applying the least square method approximation, the graphs can be substituted by the function [9]:

$$
S_{\mathrm{x}}=\frac{2 D_{\mathrm{x}} \alpha}{\alpha^{2}+\omega^{2}}
$$

Parameters $D_{\mathrm{x}}$ and $\alpha$ were adjusted using the MATLAB software and $\omega$ is used to denote frequency given in $\left[\frac{1}{s}\right]$. The analysis was performed for three velocity values 
$\left(V_{0}=10 \frac{\mathrm{m}}{\mathrm{s}}, 12 \frac{\mathrm{m}}{\mathrm{s}}, 16 \frac{\mathrm{m}}{\mathrm{s}}\right)$ and three functions obtained accordingly were approximated yielding the spectral density of irregularities of guiding strings (see Fig 5, 6, 7).

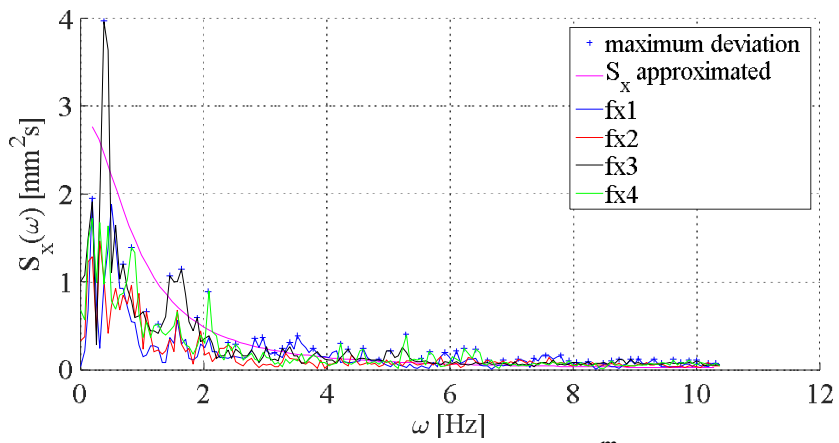

Fig 5. Spectral density function of irregularities for speed $V_{0}=10 \frac{\mathrm{m}}{\mathrm{s}}$.

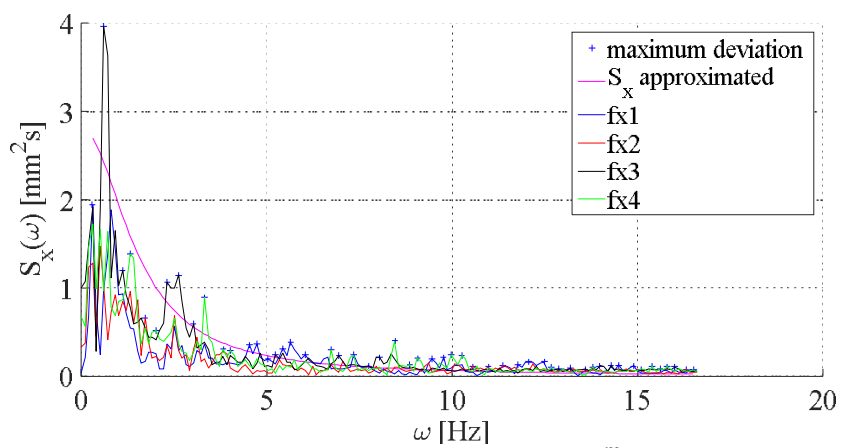

Fig 6. Spectral density function of irregularities for speed $V_{0}=12 \frac{\mathrm{m}}{\mathrm{s}}$.

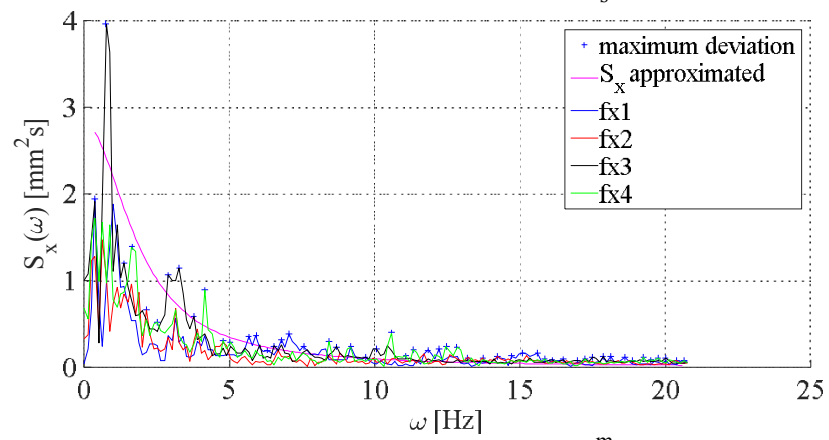

Fig 7. Spectral density function of irregularities for speed $V_{0}=16 \frac{\mathrm{m}}{\mathrm{s}}$.

Table 1. Parameters values.

\begin{tabular}{|c|c|c|c|}
\hline Velocity $V_{0}\left[\frac{\mathrm{m}}{\mathrm{s}}\right]$ & \multicolumn{2}{|c|}{ Parameters } & \multirow{2}{*}{ Formula $S_{\mathrm{x}}=\frac{2 D_{\mathrm{x}} \alpha}{\alpha^{2}+\omega^{2}}$} \\
\cline { 2 - 3 } & $\alpha\left[\frac{1}{\mathrm{~s}}\right]$ & $D_{\mathrm{x}}\left[\mathrm{mm}^{2}\right]$ & \\
\hline 10 & 0,94 & 1,32 & $S_{\mathrm{x}}(\omega)=\frac{2 D_{\mathrm{x}} \alpha}{\alpha^{2}+\omega^{2}}=\frac{2 \cdot 1,32 \cdot 0,94}{0,94^{2}+\omega^{2}}$ \\
\hline 12 & 1,50 & 2,11 & $S_{\mathrm{x}}(\omega)=\frac{2 D_{\mathrm{x}} \alpha}{\alpha^{2}+\omega^{2}}=\frac{2 \cdot 2,11 \cdot 1,5}{1,5^{2}+\omega^{2}}$ \\
\hline 16 & 1,87 & 2,64 & $S_{\mathrm{x}}(\omega)=\frac{2 D_{\mathrm{x}} \alpha}{\alpha^{2}+\omega^{2}}=\frac{2 \cdot 2,64 \cdot 1,87}{1,87^{2}+\omega^{2}}$ \\
\hline
\end{tabular}


For the steady hoisting velocity like $V_{0}=10 \frac{\mathrm{m}}{\mathrm{s}}, V_{0}=12 \frac{\mathrm{m}}{\mathrm{s}}, V_{0}=16 \frac{\mathrm{m}}{\mathrm{s}}$, the spectral density function of guide string irregularities and misalignments are written as examples in Table 1.

Plots of spectral density of guide string irregularities for the considered hoisting velocities $\left(V_{0}=10 \frac{\mathrm{m}}{\mathrm{s}}, 12 \frac{\mathrm{m}}{\mathrm{s}}, 16 \frac{\mathrm{m}}{\mathrm{s}}\right)$ are compiled in respective figures. Parameters $D_{\mathrm{x}}$ and $\alpha$ were adjusted for each hoisting velocity such that the mean square error involved in the approximation procedure should be as small as possible. As the value of spectral density function $S_{\mathrm{x}}(\omega)$ tends to increase with increasing velocity, the function used in further analyses will be that expressed as:

like in the case of $V_{0}=16 \frac{\mathrm{m}}{\mathrm{s}}$.

$$
S_{\mathrm{x}}(\omega)=\frac{9,87}{1,87^{2}+\omega^{2}}
$$

\section{Stress levels in load-bearing cables in a conveyance}

Spectral density of stresses acting in load-bearing cables of a conveyance due to irregularities and misalignments of the guide string can be derived from the formula $[3,8]$ :

$$
D_{\sigma}=\frac{3}{2} D_{\mathrm{x}} \cdot n \cdot k_{\mathrm{g}}^{2} \cdot \frac{l_{1}}{b h^{2}} \cdot I_{50}
$$

where:

$D_{\mathrm{x}}$ - parameter characterising irregularities of the guide string;

$k_{\mathrm{g}}$ - elasticity of guide string in the front plane of the skip

$l_{1}$ - midpoint of the skip height (distance between the front shoes on the skip head and those on the bottom frame)

$b, h$ - cross-section areas of the bearing cables

and

$$
\begin{gathered}
I_{50}=\frac{M_{50}}{a_{0} 4_{50}} \\
M_{50}=-a_{0}\left\{a_{5}\left[b_{1}\left(a_{3} a_{4}-a_{2} a_{5}\right)+b_{2}\left(a_{0} a_{5}-a_{1} a_{4}\right)+b_{3}\left(a_{1} a_{2}-a_{0} a_{3}\right)\right]+b_{4}\left[a_{1}\left(a_{1} a_{4}-a_{2} a_{3}\right)+a_{0}\left(a_{3}^{2}-a_{1} a_{5}\right)\right]\right\} \\
\Delta_{50}=-a_{5}\left\{a_{0}^{2} a_{5}^{2}-2 a_{0} a_{1} a_{4} a_{5}-a_{0} a_{2} a_{3} a_{5}+a_{0} a_{3}^{2} a_{4}+a_{1}^{2} a_{4}^{2}+a_{1} a_{2}^{2} a_{5}-a_{1} a_{2} a_{3} a_{4}\right\} \\
a_{0}=1 ; \quad a_{1}=2 n_{2}+n ; \quad a_{2}=1+n_{1}\left(1+2 n_{3}\right)+2 n_{2} n ; \quad a_{3}=2 n_{2}+n+n_{1} n\left(1+2 n_{3}\right) ; a_{4}=2\left(n_{1} n_{3}+n_{2} n\right) ; \\
a_{5}=2 n_{1} n_{3} n ; b_{0}=0 ; b_{1}=-n_{2}^{2} ; b_{2}=4 n_{1}^{2} n_{3}^{2} ; b_{3}=0 ; b_{4}=0 ; n_{1}=\frac{m_{\mathrm{A}}}{m_{\mathrm{g}}} ; n_{2}=\frac{h}{m_{\mathrm{g}}} \sqrt{\frac{m_{\mathrm{A}}}{k_{\mathrm{g}}}} ; n_{3}=\frac{k}{k_{\mathrm{g}}} ; \mathrm{n}=\alpha \sqrt{\frac{m_{\mathrm{A}}}{k_{\mathrm{g}}}}
\end{gathered}
$$

Since $\frac{h_{\mathrm{g}}}{h} \ll 1$, it is assumed that $h+h_{\mathrm{g}}=h$ and $h_{\mathrm{g}} \cong 0$. The remaining designations are identical to those adopted in [3].

The product $n \cdot D_{\text {x }}$ present in $\mathrm{Eq}(4)$ determines the parameters of the applied random excitations whilst the expression (5) is governed by parameters characterising the investigated object.

Variability ranges of parameters $n_{1}, n_{2}, n_{3}$ and $n$ are adjusted basing on the calculation procedure highlighted in chapters 2 and 3. Parameters used in the procedure were those of the conveyance being the object of experimental testing $[4,7]$ :

$$
m_{\mathrm{A}}=1,6 \cdot 10^{4}[\mathrm{~kg}] ; m_{\mathrm{g}}=4 \cdot 10^{3}[\mathrm{~kg}] ;
$$

Accordingly, we get : $n_{1}=\frac{m_{\mathrm{A}}}{m_{\mathrm{g}}}=\frac{1,6 \cdot 10^{4}}{4 \cdot 10^{3}}=4 ; n_{2}=\frac{h}{m_{\mathrm{g}}} \sqrt{\frac{m_{\mathrm{g}}}{k_{\mathrm{g}}}}=\frac{4000}{4 \cdot 10^{3}} \sqrt{\frac{1,6 \cdot 10^{4}}{1,54 \cdot 10^{6}}} \cong 0,10$;

$$
n_{3}=\frac{k}{k_{\mathrm{g}}}=\frac{7,5 \cdot 10^{5}}{1,54 \cdot 10^{6}} \cong 0,49 ; \mathrm{n}=\alpha \sqrt{\frac{m_{\mathrm{A}}}{k_{\mathrm{g}}}}=1,87 \sqrt{\frac{1,6 \cdot 10^{4}}{1,54 \cdot 10^{6}}} \cong 0,19
$$


Variability of $I_{50}$ depending on $n_{1}=\frac{m_{\mathrm{A}}}{m_{\mathrm{g}}}$ for $n_{2}=0,10, n_{3}=0,49$ oraz $n=0,19$ is plotted in Fig. 8.

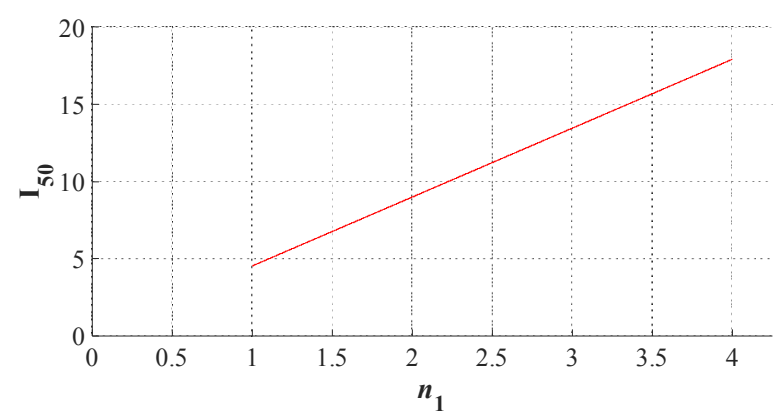

Fig. 8. Diagram of $I_{50}$ depending on $n_{1}$.

The value of $I_{50}$ read out from Fig. 8 and obtained for the skip parameters listed above is found to be $I_{50}=18$.

In accordance with (4), spectral density of stresses in load-bearing cables due to irregularities and misalignment of the guide string becomes:

$$
D_{\sigma}=\frac{3}{2} \cdot 2,64 \cdot 10^{-6} \cdot 0,19 \cdot 1,57^{2} \cdot 10^{12} \cdot \frac{16}{0,03 \cdot 0,25^{2}} \cdot 18=0,274\left[\mathrm{MPa}^{2}\right]
$$

Standard deviation of maximal stresses acting in load-bearing cables in the case analysed in this study is derived from the formula:

\section{Conclusions}

$$
\sigma\left(D_{\sigma}\right)=\sqrt{D_{\sigma}}=\sqrt{0,274} \cong 0,52[\mathrm{MPa}]
$$

Predicted values of standard deviations of maximal stresses acting in load-bearing cables in a skip are decidedly lower than those measured experimentally [4, 6]. Such significant discrepancy (30-fold) can be attributable to the fact that theoretical considerations relied on spectral densities of irregularities and misalignments of the guide strings reported in [6], and derived from normative reference documents [9, 10] having relevance to conveyances in hoisting installations. Predicted data given in this study, based on irregularities and misalignments readouts in real conditions [11], are decidedly higher, yet the discrepancy between the theoretical and experimental results is still unacceptable.

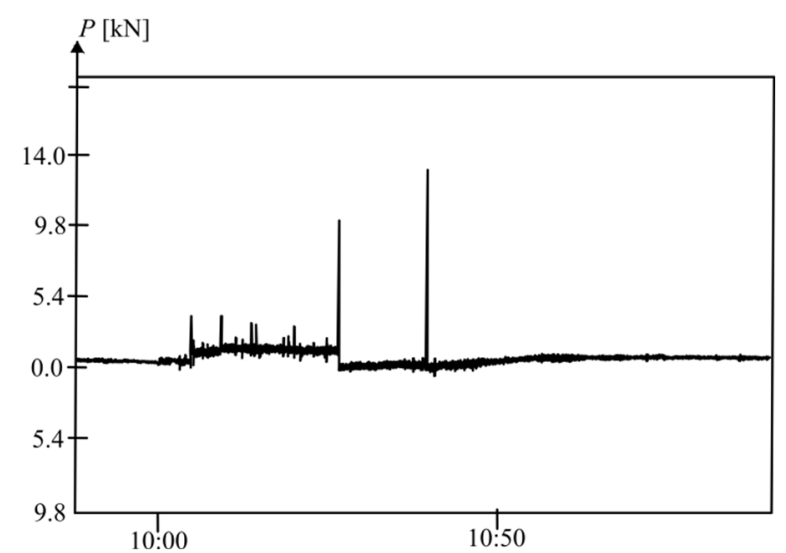

Fig. 9. Diagram of the conveyance -shaft steelwork interaction force [7]. 
Comparing and contrasting the experimental and predicted data will have its merits as long as all assumptions and conditions underpinning the dynamic analysis are fully satisfied. Actually, results of an experiment performed on the same object yet limited to measurements of the conveyance-shaft steelwork interaction forces summarised in [7] indicate the occurrence of impact effects in the systems whilst in fact the conveyance will run smoothly on the guide rails along short hoistway sections only. This behaviour is revealed in plots of conveyance-shaft steelwork interactions shown in Fig. 9 [7], where the peaks represent forces arising due to impacts between the skip and shaft steelwork.

It appears that when hoist operators attempt to distance the guides from the guide shoes, they do so for their own reasons. In order that theoretical results could be reliably verified experimentally[12], the conveyance needs to remain in contact with the guides, which is a requirement stipulated in relevant mining regulations.

\section{References}

1. S. Wolny, Interactions in Mechanical Systems Due to Random Inputs on the example of a Mine Hoist, International Education and Research Journal, vol. 1. iss.5,s.72-76 (2015)

2. S. Wolny, Displacements in Mechanical System Due to Random Inputs in a Mine Hoist Installation, Engineering Transactions. Engng. Trans. 65,3,p. 513-522 (2017)

3. S. Wolny, Loads experienced by load-bearing components of mine hoist installations due to random irregularities and misalignments of the guide string, Journal of Machine Construction and Maintenance, vol. 110, iss. 3,p.79-86 (2018)

4. S. Wolny et al., Developing critiria for assessing the supporting structure skip hopper of hoist installation in the terms of extending the period of safe operation, Scientific an reasearch work, Department of Strength of Materials and Structures, AGH, Cracow (2003)

5. S. Wolny et al., Development of multi-option modernization of mining cage lift $R$ VII, Scientific and research work, Department of Strength and Fatigue of Materials and Structures, AGH, Cracow (2011)

6. S. Wolny, Experimental verification of the state of stress in local Bering cables in a hosting installation due to random misalignments and irregularities of the guide strings, (Article submitted for publication in Archives of Mining Sciences) (2018)

7. D. Bańdo, The impast of selected operating parameters on the strength of structural elements of the skip hopper, PhD thesis, AGH, WIMiR, Depatrment of Strength of Materials and Structures, Cracow (2006)

8. R. Gutowski, W. A. Swietlicki, Dynamics and vibrations of mechanical systems, PWN, Warsaw (1986)

9. S. Kawulok, The influence of the shaft steelwork on mechanics of guiding skip hopper, GIG works, Katowice (1989)

10. Mining Regulation: The Council of Ministers regulation of 30 April 2004 in the admission of using in mining plants (Dz.U. No. 99,item 1003 of 2005 No. 80, item 695 and of 2007 No. 249, item 1853, p.1.2 skip hopper).

11. F. Matachowski, Developing design criteria of selected skip hopper elements, $\mathrm{PhD}$ thesis, AGH, WIMiR, KWZMiK, Cracow (2011)

12. M. Szot, Influence of irregularities of stiff guidance of shaft conveyances on the change of hoisting rope loading- methodology of testing, measuring equipment, Mining and Environment, Quarterly 4, Central Mining Institute, Katowice (2003) 